\title{
Study on Entrance Lighting and Traffic Safety of Expressway Tunnel
}

\author{
Ran Li \\ China Academy of Transportation Sciences, Beijing 100029, China \\ liranxz@126.com
}

Keywords: Tunnel entrance lighting, driving safety, changing rate of pupil, luminance reduction coefficient.

\begin{abstract}
When the driver of the expressway enters the tunnel, if the entrance lighting brightness settings unreasonable, the driver experienced drastic changes from the high-brightness environment outside the tunnel to the low-brightness environment in the tunnel, easily lead to driver visual obstacles, so that the driver can not see the front road conditions, which is leading to the tunnel entrance more major traffic accidents. Ten typical highway tunnels with a speed limit of $60 \mathrm{~km} / \mathrm{h}$ in the mountainous area is used as the test environment. Using the experimental equipment such as illuminometer and eye tracker, the driving factors of the small passenger car are collected and analyzed. Acquisition and analysis data of the change rate of pupil of the car driver under the the different luminance reduction coefficient (inside and outside the tunnel brightness ratio).Regression model is developed and according to the safety threshold, changing rate of pupil was $20 \%$, we can obtain the safety luminance reduction coefficient, expressway tunnel luminance reduction coefficient standard with a speed limit of $60 \mathrm{~km} / \mathrm{h}$ was 0.031 . This is higher than Chinese standard. The conclusion of the study is of reference value to the theory and practice of highway entrance lighting design and operation management.
\end{abstract}

\section{Introduction}

Because of the closeness of its structural space, so that the difference between the brightness inside and outside the tunnel, especially in the clear daytime, resulting in the driver visual adaptation difficulties when into the tunnel. It is difficult to identify the front road, traffic and environmental conditions. Due to the narrow space of tunnel, escape and rescue are very difficult, can easily lead to the major accidents. During January 2010 to December, the number of traffic accidents in a mountain freeway tunnel threshold zone accounted for $54.5 \%$ of the total number of the tunnel traffic accidents. The Norway Highway Administration investigations have shown that the tunnel traffic accident rate was in a interval distribution, the tunnel threshold zone accounted for $63.7 \%$ of the total number of the tunnel traffic accidents, which is accident-prone section [1]. Therefore, the tunnel entrance lighting should be the appropriate brightness of the enhanced lighting, reduce the difference between the brightness inside and outside the tunnel, to create a safe tunnel lighting conditions, tunnel entrance lighting on the driving safety of the tunnel has become an urgent need to solve the key issues one.

Currently almost countries include China use the index of luminance reduction coefficient to design tunnel entrance lighting and the standard value of China is developed with reference to Japan's. The criterion of tunnel luminance reduction coefficient in China is shown in Tab.1 [2]. Compare to other country, the luminance reduction coefficient in China is lower than other country, such as in U.K., Norway and CIE (International Commission on Illumination) in Tab.2 [3, 4, 5]. The standard of the reduction factor of the tunnel in China does not take into account the geographical characteristics, and the visual characteristics of the Chinese driver during the dynamic driving process, and should be studied in a reasonable way. 
Table 1 Standard of luminance reduction coefficient

\begin{tabular}{|c|c|c|c|c|c|c|}
\hline \multirow{2}{*}{$\begin{array}{c}\text { Design Traffic Volume N } \\
\text { veh/h }\end{array}$} & \multicolumn{5}{|c|}{ luminance reduction coefficient } \\
\cline { 2 - 7 } & \multicolumn{5}{|c|}{$\begin{array}{c}\text { Design Speed } \\
\mathrm{km} / \mathrm{h}\end{array}$} \\
\hline One-way & Two-way & 120 & 100 & 80 & 60 & $20 \sim 40$ \\
\hline$\geq 1200$ & $\geq 650$ & 0.070 & 0.045 & 0.035 & 0.022 & 0.012 \\
\hline$\leq 350$ & $\leq 180$ & 0.050 & 0.035 & 0.025 & 0.015 & 0.010 \\
\hline
\end{tabular}

Note: When the traffic volume is at inter of above two volumes, you can take interpolation.

Table 2 Luminance Reduction Coefficient in Different Countries

\begin{tabular}{|c|c|c|c|c|}
\hline \multirow[b]{2}{*}{ Standards Classification } & \multicolumn{2}{|c|}{ Traffic Flow } & \multirow{2}{*}{$\begin{array}{c}\text { Speed } \\
\mathrm{km} / \mathrm{h}\end{array}$} & \multirow[b]{2}{*}{ Luminance Reduction Coefficient } \\
\hline & $\begin{array}{l}\text { AADT } \\
\text { veh/d }\end{array}$ & veh/(h·lane & & \\
\hline \multirow{3}{*}{ CIE } & \multirow{3}{*}{ - } & \multirow{3}{*}{ - } & 120 & 0.10 \\
\hline & & & 80 & 0.06 \\
\hline & & & 60 & 0.05 \\
\hline \multirow{5}{*}{ Norway } & $>7500$ & \multirow{5}{*}{ - } & - & 0.05 \\
\hline & \multirow{2}{*}{$5000 \sim 7500$} & & 80 & 0.05 \\
\hline & & & 50 & 0.025 \\
\hline & \multirow{2}{*}{$2500 \sim 5000$} & & 80 & 0.03 \\
\hline & & & 50 & 0.015 \\
\hline \multirow{9}{*}{ U.K. } & \multirow{9}{*}{ - } & \multirow{3}{*}{$>1500$} & 110 & 0.07 \\
\hline & & & $80 \sim 100$ & 0.05 \\
\hline & & & \begin{tabular}{|l|}
$48 \sim 65$ \\
\end{tabular} & 0.04 \\
\hline & & \multirow{3}{*}{$500 \sim 1500$} & 110 & 0.05 \\
\hline & & & $80 \sim 100$ & 0.04 \\
\hline & & & $48 \sim 65$ & 0.03 \\
\hline & & \multirow{3}{*}{$<500$} & 110 & - \\
\hline & & & $80 \sim 100$ & - \\
\hline & & & \begin{tabular}{|l|}
$48 \sim 65$ \\
\end{tabular} & - \\
\hline
\end{tabular}

Note: The number of vehicles per hour per lane can be calculated by dividing peak hour value by the total number of lanes.

Tunnel entrance lighting research abroad started earlier. D.A.Schreuder created a tunnel experimental model which for foundation experiment of tunnel lighting in the early 60s of the last century and made a perceived probability experiments with a model of small target. This study got the functional relationship between tunnel standard section luminance and threshold zone luminance [6]. Nakamichi and Narisada. et al. considered the functional relationship between the background brightness of visual recognition target and the time observer start pay attention to the tunnel entrance and studied the lighting of tunnel by experiments [6].Based on studies of above researchers, such as D.A.Schreuder, Nakamichi and Narisada, et al., CIE presented a methodology to evaluate the lighting of tunnel threshold zone that lighting standard must can make driver see an object on the pavement when driver is in front of the tunnel entrance and close tunnel, the distance between driver and object is called the stopping distance at a certain design speed[5]. Thompson assessed the effect of contrast ratio on the judgment of speed. It was found that reduction in contrast ratio leads to an underestimation of driving speed. The threshold of speed underestimation depends on the spatial frequency of visual information [7]. Buchner et al. Investigated the effect of illumination on the judgmentof distance between vehicles. The results showed that reduced illumination in tunnels leads to increased perceived distance of drivers [8]. Zhigang Du, Xiaodong Pan and Xuebin Guo. testified the relationship of power function between the driver pupil area and tunnel entrance or exit illumination in tunnel departing from the entrance or exit $50 \mathrm{~m}$ range by using illuminance meter, acceleration acquisition instrument, dynamic eye tracker to test driver pupil area at tunnel entrance and exit and analysis. This method developed the change speed of driver pupil area to evaluate traffic 
safety at tunnel entrance and exit. Statistical analysis showed that the safe critical velocity value of driver pupil area change is in $-6 \mathrm{~mm} 2 / \mathrm{s}$ to $4 \mathrm{~mm} 2 / \mathrm{s}$ and concluded a calculation model of tunnel entrance and exit lighting based on driver visual adaptation [9]. Zhigang Du, Xiaodong Pan and Zhen Yang used dynamic eye tracker to study the driver's papillary changes at freeway tunnel entrance and exit and evaluated the visual load level and operation safety based on the field test and the visual turbulence in the light and dark adaptation. They got results that when the operation speed is under $85 \mathrm{~km} / \mathrm{h}$, the driver can get a good visual comfort level and the stopping distance at the freeway tunnel entrance and exit is higher than the existing norms, an increase of 20 to 30m [10]. Xiaodong Pan, et al. presented a analyze methodology to determine the correlation of driving visual impairment, visual comfort and visual load level by using a new index value which is called the visual load index of the pupil largest transient speed based on a large number experiments of the moving vision point on the fields. Therefore it is advisable that the improvable range of visual environment under different limited speed at the day or night and some improvement measures [11]. Qingwen Zhang, et al. used dynamic eye tracker to monitor the information of visual tracking by field experiments of multiple tunnel entrances and evaluate the quality of the tunnel entrance lighting by statistics and analysis these information parameters of the small target gaze time, gaze numbers and gaze duration using dynamic video analysis software [12].

According to above analysis, there are different standards in different country about light of tunnel luminance reduction coefficient, especially the standard of China need further study. About tunnel entrance lighting safety quantitative study method, there are change speed of driver pupil, driving visual impairment time, gaze time and numbers of vision, etc. This paper attempts to study the influence of different luminance reduction coefficient on the driving safety visual at the tunnel entrance section. The paper analyzes the influence of different luminance reduction coefficient on the pupil area change of the driver when entering the tunnel, establishes the changing rate of pupil. The relationship model is used to determine the value of the luminance reduction coefficient that ensures the safety of the driver in tunnel traffic.

\section{Pupil Area Change and Traffic Safety}

Pupil changing is one important index of human physical and psychological. When people is in psychological activities, his pupil size changes a lot and pupil size increased means greater workload or mental effort in cognitive activity. The size of the pupil is jointly controlled by pupillary sphincter and pupillary dilator muscle. Pupillary sphincter is controlled by parasympathetic nerve of oculomotor nerve and pupillary sphincter shrinks causes the pupil size decreased. Pupillary dilator muscle is controlled by sympathetic nerve and pupillary dilator muscle shrinks causes the pupil diameter increased.

Physical reactions is related to sympathetic nerve function. Major function of sympathetic nerve make pupil size increased, accelerating the heartbeat, shrinking skin and splanchnic vessel, widening the coronary arteries and elevating blood pressure. When facing threat, emergency situations, or felling exciting, sympathetic nerve activity plays a major role; the function of parasympathetic nerve is to save energy, slow down the body's reaction, pupil size decreased to reduce irritation, slow down the heartbeat, lower blood pressure. When the body is in relaxing state, parasympathetic nerve is positive. Therefore, the change of pupil can reflect unsafe and uncomfortable state of people's physiological and psychological that the drivers encountered in the process of driving [13].In this study, we propose "changing rate of pupil(CRP)" index to quantify the level of driving safety and the index can be represented as such

$$
C R P=\left(e_{i+1}-e_{i}\right) / e_{i}
$$

Where $\mathrm{e}=$ driver's pupil area, $\mathrm{px} ; \mathrm{i}=$ Measuring time of the pupil area, $\mathrm{i}+1$ is $\mathrm{i}$ 's next time.

According to our research achievement, thresholds of CRP when the driver is in safe state, more dangerous state or very dangerous state, are as following [14]:

(1) Safe state: CRP $<20 \%$; 
(2) More dangerous state: $20 \% \leqslant \mathrm{CRP} \leqslant 40 \%$;

(3) Very dangerous state: $\operatorname{crp}>40 \%$.

In free-flow traffic and good alignment condition, the driver's pupil area changes caused by different tunnels lighting conditions when driving into tunnel lighting access zone and threshold zone.Fig. 1 shows the car driver's crp area when driving into tunnel lighting impacting area under free-flow traffic condition at a certain operation speed. Driver's crp area stays stable when driving through normal segment of tunnel lighting impacting area except for individual outliers. In tunnel access zone, there is a great difference between the natural luminous illumination and tunnel illumination. Driver see a "black hole", it makes the driver can't see the information about the road, traffic and environment conditions, be called "black-hole effect", and makes driver feels nervous, the pupil area appears a sharp increase and crp emerges the value which is more than $20 \%$.

The driver who had passed different changes in illumination can't rapidly adapt to the environment in vision instantly when he drive into the tunnel or threshold zone. That will lead to problem of dark adaptation and needs a period of time to adapt and could see the driving conditions. During these times, the driver feels nervous and CRP fluctuates obviously or CRP increases and the value even increases up to $20 \%$. The higher speed, the longer the adaptation time is.

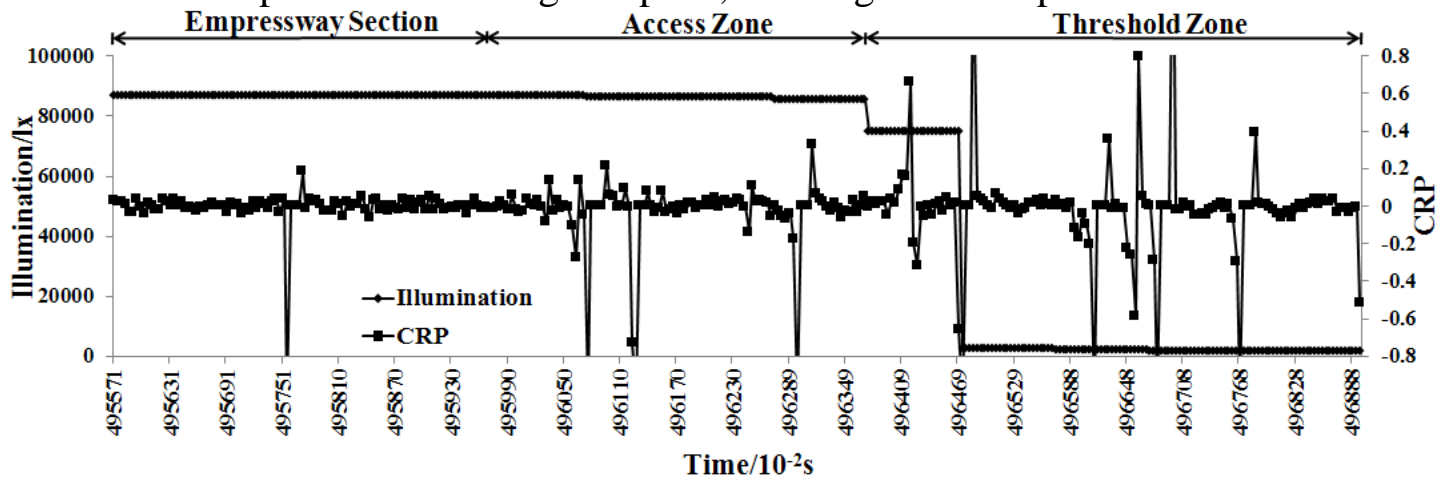

Fig.1 Changing law of drivers' CRP in tunnel entrance section

Unreasonable tunnel lighting can cause driver's nervous feeling and increasing CRP. This will impact driving safety. In a certain tunnel lighting conditions, the higher speed, the more nervous the driver feels and then CRP increases. The main purpose of this study is to examine the interrelationship among them. More specifically, we attempt to demonstrate.

\section{Experiment Design}

The purpose of this study is to analyze the relationship among driver CRP under different brightness condition in expressway. To avoid potential biases resulted by the presence of other vehicles, two-lane one way expressways with very low volume were selected for this research. All partial data affected by the presence of other vehicles was removed to maintain the most consistent experimental conditions. There are 10 tunnels with straight line threshold zone which illuminations are difference and design speed is $80 \mathrm{~km} / \mathrm{h}$, limited speed is $60 \mathrm{~km} / \mathrm{h}$. The length of each tunnel is between $973 \mathrm{~m}$ and $12330 \mathrm{~m}$. Drivers which 6 healthy and normal drivers with normal visions were selected randomly in the test site to processed real vehicle test. The tested vehicle is 1.8T Roewe 750 .

\section{Experiment Instrument}

An instrumented vehicle equipped with an dynamic eye tracker system(iView $\mathrm{X}$ HED, SensoMotoric Instruments, Inc., Germany) which used for recording the experimenter's position of eye fixation points, pupil area, etc. The illumination-meter (CL-200A, Konica Minolta Holdings, Inc., Japan) were used for collecting the illumination values dynamically during the test drive in and out of the tunnel. At the same time, the testers sit beside with tested driver record the test start and end time, 
stake number, overtaking, overtaken by other vehicles and other unusual driving behavior of the tested drivers.

\section{Data Analysis}

Excluding the sample data which affect the driver physiological and psychological and driving behavior characteristics in non-tunnel lighting conditions in the 50 groups of sample obtained in the experiments, and get 30 groups of samples in the effective test of car drivers under free-flow traffic conditions.

Based on the field useful data, it was found that empirically the variation characteristics and the representative value of the changing rate of driver's pupil and luminance reduction coefficient when subjects drive into the expressway tunnel threshold zone to arrive to the end of the tunnel threshold zone, using the maximum fluctuation change rate of the pupil from the driver's pupil begin to increase as the eigenvalues of the CRP, using the minimum ratio of the luminance of two consecutive points from the driver's pupil begin to increase as the eigenvalues of the luminance reduction coefficient (LRC). Through analysis to the driver's driving demand in the tunnel threshold zone lighting, we found that CRP and LRC exist different degree of correlation. We make the further quantitative correlation analysis.

Relationship between CRP and LRC.The relationship between CRP and reciprocal of LRC by 15 groups of samples data drawing a scatter diagram at the tunnel threshold zone lighting is shown in Fig.2. We can notice driver's CRP and the reciprocal of tunnel threshold zone LRC distribution. It can be notice that when subjects drive through the tunne threshold zone lighting, their correlation is positive significant.

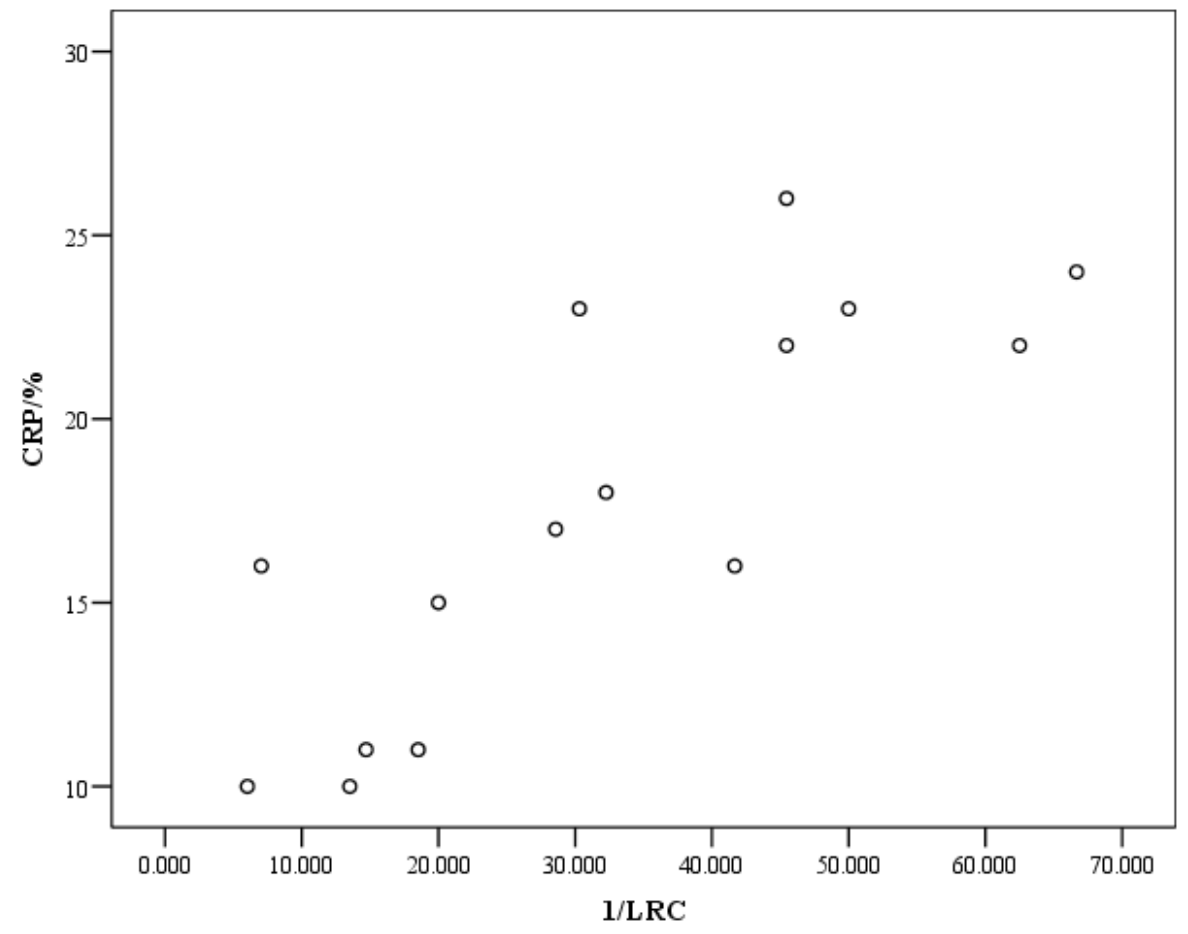

Fig.2 Relationship between CRP and the reciprocal of LRC

\section{Modeling}

We employed a regression technique to examine the relationship among CRP and LRC . By using SPSS(13.0), we got relationship model among CRP and LRC and represented as such. 


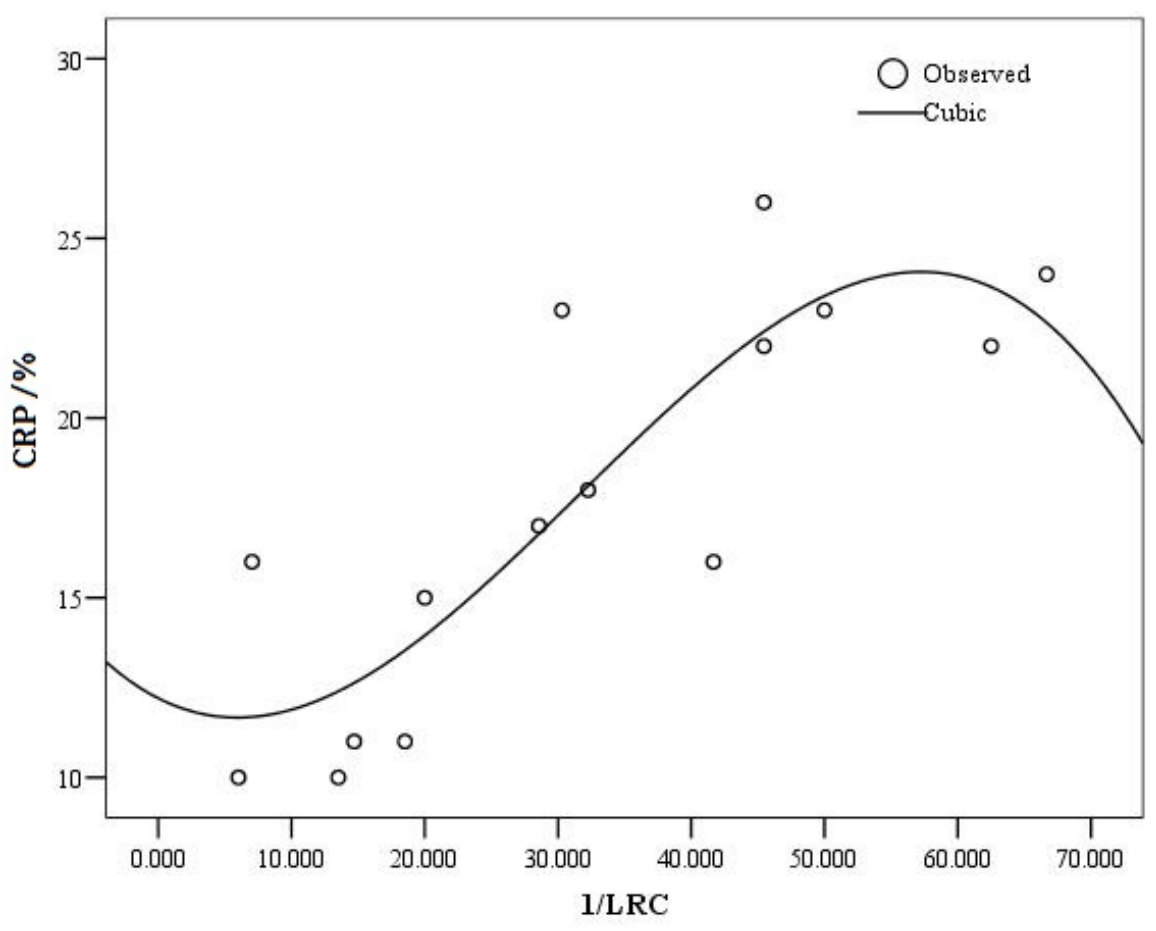

Fig.3 Curve of model fitting

$$
C R P=-0.188 / L R C+0.017 / L R C^{2}+0.000184 / L R C^{3}+12.205
$$

The model's correlation was statistically significant $\left(\mathrm{R}^{2}=0.729\right)$, and the application scope is: LRC $\in[0.015,0.167]$ in free flow traffic conditions and limited speed is $60 \mathrm{~km} / \mathrm{h}$. The model fitted well by significance testing and residual analyzing.

\section{Safety Analysis at The Threshold Zone Lighting}

According to the regression model(1) of driver's CRP in the tunnel threshold zone, we can put the lowest threshold value(20\%) which meets driver's safety into the model, we can calculate the least standard value which meets driver's safety at threshold zone lighting under speed limit $60 \mathrm{~km} / \mathrm{h}$ conditions.

Such as when expressway tunnel speed limit is $60 \mathrm{~km} / \mathrm{h}$, we can calculate the safety threshold of LRC in the tunnel threshold zone is 0.032. Compare the calculation result with the rule of Specifications for Design of Ventilation and Lighting of Highway Tunnel in China, we can find that it is obvious higher than 0.015 when traffic volume is less than or equal to $350 \mathrm{veh} / \mathrm{h}$ and 0.022 when traffic volume is equal or greater than $1200 \mathrm{veh} / \mathrm{h}$,but the value is close to the standards of other countries.

\section{Conclusion}

The research presented in this paper provides a practical method to evaluate driver's safety caused by differences between inside and outside tunnel lighting. By field experiments to examine changing rate of driver's pupil and luminance reduction coefficient under speed limit $60 \mathrm{~km} / \mathrm{h}$ at two mountainous expressway with 10 tunnel entrance sections in china, we developed a regression model. This model can be applied to get the luminance reduction coefficient standard, need of driver's safety at expressway tunnel entrance and applied to both existing expressway assessments and new expressway designs. The methodology also supports research of the other road tunnels and other zones. 


\section{References}

[1] Vauquelin, O., Megret, O. (2002), Smoke extraction experiments in case of fire in a tunnel, Fire Safety Journal. 37(5):525-533.

[2] JTG/T D70/2-01-2014, Guidelines for Design of Lighting of Highway Tunnels. Recommended Industry Standards of the People’s Republic of China, 2014.

[3] British Standards Institution., Code of practice for the design of road lighting-Lighting of tunnels”, BS 5489-2-2003, 2003.

[4] Norwegian Public Roads Administration, Road Tunnels, Norwegian: NPRA Printing Center, 2004.

[5] International Commission on Illumination, Guide for the Lighting of Road Tunnels and Underpasses, 2004.

[6] International Commission on Illumination, Tunnel Entrance Lighting: A survey of fundamentals for determining the luminance in the threshold zone, 1984.

[7] Thompson, P., Velocity after-effects: the effects of adaptation to moving stimuli on the perception of subsequently seen moving stimuli. Vision Res. 21 (3) (1981)337-345.

[8] Buchner, A., Brandt, M., Bell, R., Weise, J., Car backlight position and fog density bias observer-car distance estimates and time-to-collision judgments. Hum. Fact. J. Hum. Fact. Ergon. Soc. 48 (2) (2006) 300-317.

[9] Du, Z. G., Pang, X. D., Guo, X.B.., Experimental studies of visual adaptation on driving through freeway tunnel's entrance and exit, Journal of Harbin Institute of Technology. 39 (12) (2007)1998-2001.

[10] Du, Z. G., Pang, X. D., Yang, Z., Guo, X.B.. , Research on Visual Turbulence and Driving Safety of Freeway Tunnel Entrance and Exit, China Journal of Highway and Transport, 20(5) (2007)101-105.

[11] Pang, X. D., Song, Y. C., Yang, Z., Zhang, G. S.. , Visual Environment Improving Scope at Entrance and Exit of Highway Tunnel Based on Visual Load, Journal of Tongji University(Natural Science).37(6) (2009)777-780.

[12] Zhang, Q. W., Xu, Y., Hu, Y. K. , Highway Tunnel Entrance Illumination Measurement Method Based on Physiological and Psychological Effects, Illuminating Engineering Journal, 23(2) (2012)8-14.

[13] LI Yong, YIN Guo-en, CHEN Yan-li, The Regulating Effect of Fatigue and Mental Load on Pupil Size in Text Reading, Studies of Psychology and Behavior.2(3)(2004)545-548.

[14] Zhao, L, Two-lane Highway Alignment Research Based on Driver's Psychological and Physiological Reaction, Beijing University of Technology, Beijing, 2008. 